\title{
An Act of Futility, or Pascal's Wager?
}

\section{Hutchins $\mathrm{AM}^{* 1}$ and Winham $\mathrm{DM}^{2}$}

Pavía Hospital, A Private Hospital in San Juan, USA

*Corresponding author: Enrique Vázquez-Quintana, MD, FACS, Urb. El Remanso, F-15 Corriente St. San Juan, PR 00926-6108, USA, Tel: 7874620658; Email: evazquezmd@gmail. com

\section{Case Report}

Volume 4 Issue 1

Received Date: February 21, 2020

Published Date: March 11, 2020

DOI: $10.23880 /$ cprj-16000120

\section{Abstract}

Futility is defined as something unimportant, useless, ineffective, without value or purpose, frivolousness, unable to solve problems. The definition of futility is clear and simple; the difficultly is its clinical application in the management of complex cases. The implications are different when dealing with medical or surgical patients. It is radically different when the decision of not doing any other efforts for the treatment of intractable heart failure or disseminated carcinomatosis or when we are dealing with surgical patients whose diseases carry very low opportunity of survival or of improvement in his general wellbeing. It is in the medical cases that the strategy of hanging the Crepe is used when trying to communicate to the relatives the gravity of their beloved ones illness. At times the physicians exaggerate the seriousness of the disease to lessen the suffering if eventually the patient dies. Crepe is a black piece of cloth forming a bow that used to be placed in the front door of the house to indicate the death and mourning of the relatives. This custom has been abandoned in almost all countries. Physicians undertake the utmost efforts in trying to preserve the life of their patients as well as to provide quality of life no matter the economic circumstances. In recent years I had two patients who were so sick that their condition gave the impression that their lives were close to an end. Nevertheless, after an operation deemed by some other physicians as futile, both patients recovered and are leading a normal lives for the past six and five years respectively.

Keywords: Renal disease; Ulcer disease; Parathyroid scan; Echocardiography; Serum calcium; Hypercalclemia

Abbreviations: PTH: Parathyroid Hormone; SPECT: Single-Photon Emission Computed Tomography; MRI: Magnetic Resonance Imaging; CT: Computed Tomography; BUN: Blood Urea Nitrogen.

\section{Introduction}

\section{Case 1: Summary of First Patient}

This is an 81 year old female admitted to the Pavía Hospital in San Juan, PR on June 4, 2009. She was complaining of severe bone pain, mainly on her back. She has been suffering from End Stage Renal Disease for the last seven years, being dialyzed three times per week. She also suffers from hypertension, depression and peptic ulcer disease. She had had surgery of the left thyroid lobe; date and procedure done were unknown. She was admitted to rule out osteomyelitis of the thoracic or lumbar spine. Her husband died on June 8, 2009, at Pavia Hospital while she was hospitalized and a son had died one year earlier from a tumor. Her laboratories on admission were as follows: $\mathrm{Hb} 14.5 \mathrm{~g} / \mathrm{dl}$, Hct $45.2 \%$, platelets 428,000 10/ul, Calcium $16.6 \mathrm{mg} / \mathrm{dl}$, albumin $3.2 \mathrm{~g} / \mathrm{dl}$, FBS $103 \mathrm{mgs} / \mathrm{dl}$, creatinine $2.85 \mathrm{mg} / \mathrm{dl}$. Ionized calcium was 7.5 $\mathrm{mg} / \mathrm{dl}$. PTH-intact was as high as $3301 \mathrm{pg} / \mathrm{ml}$.

Parathyroid Scan with SPECT on June 18, 2009, revealed a Parathyroid adenoma at the upper pole of the left lobe. The Thyroid ultrasound on June 20,2009, was interpreted as a possible parathyroid adenoma, solid lesion 2.44 X 1.26 X 1.60 $\mathrm{cm}$ at lower left neck region. Thoracic spine MRI, Gallium 


\section{Clinical Pathology \& Research Journal}

scan with SPECT showed no evidence of osteomyelitis of the spine. CT of the head was normal. The Chest $\mathrm{x}$-rays on June 4, 2009, showed cardiomegaly and clear lungs. Echocardiography on June 2, 2009, revealed left ventricular hypertrophy, competent valves, the $\mathrm{EF} 65 \%$ and trace pericardial effusion. A left upper extremity arterial Doppler examination on April 7, 2009, revealed a patent arteriovenous fistula. The patient was seen in consultation by the nephrologist, infectious disease, cardiologist, physiatrist, pneumologist and psychiatrist. She was treated with Haldol. She had severe bony pains not relieved with analgesics. She was having disorientation, and depression. She had no history of renal lithiasis.

All the consultants were not too enthusiastic in recommending surgery to this patient. Even her cardiologist was against doing surgery on this lady. Her cardiologist stated in the chart that: "I talked to the surgeon and internist indicating that although the operation is indicated, it carries an incredible risk and will not relieve her pain syndrome acutely, she might not tolerate anesthesia and the postoperative complications that may occur. I talked with her daughters and exposed my clinical considerations. No active cardiovascular problem. I will see her as needed". The patient was treated with Sensipar until the day of operation. On June 22, 2009, the neck was explored. A large left upper parathyroid adenoma was removed. The left lower parathyroid gland was not found. The operation lasted 35 minutes. The pathology revealed a left superior parathyroid adenoma weighing 5 grams, and measuring $2.8 \times 2 \times 1.5 \mathrm{~cm}$.

The serum calcium decreased progressively, PTH-intact decreased to normal levels. She improved slowly and by her fourth postoperative day she started to recover mentally and ambulation was started. She started to eat normally. The back pains disappeared. Following the operation the calcium decreased to $10.9 \mathrm{mg} / \mathrm{dl}$, PTH- intact was $95.3 \mathrm{pg} / \mathrm{dl}$ and serum albumin was $2.4 \mathrm{~g} / \mathrm{dl}$. The patient was discharged on July 6, 2009. She continues hemo-dialysis three times a week. She is leading an active life, with good quality of life six years after her operation. Sadly she died in 2018. But the operation provided her of nine years of extra life.

\section{Case 2: Summary of Second Patient}

This is a 69 year old female with history of hypercalclemia of unknown duration. She was admitted to the HIMA Hospital in Caguas, PR. She was bedridden with disorientation, a large sacral decubitus ulcer and pneumonia. She was treated with antibiotics and hydration. Although she recovered from the pneumonia, she continued bedridden and disoriented. Neurological evaluation discarded the presence of Alzheimer disease. She has nephrolithiasis, osteoporosis and renal insufficiency. She also suffers from hypertension since age 35 and is receiving Cardura, Avapro and Norvasc. She had a myocardial infarct in 2004. She was transferred to the Pavía Hospital in San Juan, PR, on June 21, 2010. The patient was totally disoriented, unable to express herself, with a large sacral decubitus ulcer. She was recovering from pneumonia; she was evaluated by the pneumologist, cardiologist, nephrologist and infectious disease specialists. Physical therapy was started. Laboratory tests: the calcium was $11.2 \mathrm{mg} / \mathrm{dl}$, PTH was $269 \mathrm{pg} / \mathrm{ml}$, and the Creatinine Clearance was $41.59 \mathrm{ml} / \mathrm{min}$. A thyroid sonogram done on May 14, 2010, showed a solid mass at the left posterior region measuring $2.6 \times 1.2 \mathrm{~cm}$. The Sestamibi scan with SPECT on May 11, 2010, revealed an abnormal parathyroid tissue at the region of the left thyroid gland suspicious for a parathyroid adenoma. Neck CT on May 21, 2010, showed a solid circumscribed mass posterior to the left lobe of the thyroid, compatible with a parathyroid adenoma. Brain CT on May 18, 2010, revealed a left temporo-parietal defect consistent with a hemorrhagic infarction. Echocardiogram revealed a low ejection fraction of $40 \%$.

The neck was explored on June 24, 2010. A large left upper parathyroid adenoma was removed. The pathology revealed a parathyroid adenoma measuring 4.2 X $2.3 \times 1.0$ $\mathrm{cm}$. No lesion found on the lower parathyroid region. She had a slow improvement, but in one month she recovered her mental abilities, was able to recognize her relatives who were taking care of her. The serum calcium levels decreased gradually as well as her PTH-intact levels. She was discharged home on July 21, 2010. She was seen in my office on August 13, 2010. She was mentally clear, oriented, talkative. The sacral ulcer was almost healed. The PTH intact was $28.1 \mathrm{pg} /$ $\mathrm{ml}$ on August 23, 2010. Two month later her calcium was 9.10, $9.70 \mathrm{mg} / \mathrm{dl}$, phosphorus $2.80 \mathrm{mg} / \mathrm{dl}$. Creatinine $2.76 \mathrm{mg} / \mathrm{dl}$, BUN $61 \mathrm{mg} / \mathrm{dl}$, PTH-intact $45 \mathrm{pg} / \mathrm{dl}$. A Parathyroid scan with SPECT on October 1, 2010, was negative. Renal Sonogram on October 2, 2010 showed renal parenchymal disease and an atheromatous and tortuous aorta with a $2.0 \mathrm{~cm}$ sacular aneurysm. A CT of the brain on October 9, 2010, revealed involutional changes throughout the brain and white matter changes probably representing ischemia. On April 2011, she developed bradycardia and was diagnosed as having Sick Sinus Syndrome. On April 11, 2011, a pacemaker was implanted. During that admission the calcium was 9.1, 9.0 $\mathrm{mg} / \mathrm{dl}$, alkaline phosphatase $89 \mathrm{mg} / \mathrm{dl}$, creatinine $1.69 \mathrm{mg} /$ dl, BUN $25 \mathrm{mg} / \mathrm{dl}$. The CT scan of the brain on April 11, 2011, was normal.

\section{Discussion}

Neuropsychiatric symptoms are reported in about $5 \%$ of patients with severe hypercalcemia. Surgery is the accepted modality of treatment for these patients since medical treatment is not effective and does not solve the cause of 


\section{Clinical Pathology \& Research Journal}

the hypercalcemia [1-3]. These two patients presented with severe neuropsychiatric symptoms and signs secondary to the hypercalcemia. Both patients were classified as high risk patients. The non-surgical specialists were not too enthusiastic as to recommend surgery on these patients. On the other hand, as the surgeon consulted to manage these patients, I was convinced that the only chance for them to survive was to explore the neck and remove the parathyroid adenoma. The surgical management of these patients for almost all the physicians was qualified as an act of futility.

Multiple articles in the literature address the term of futility. Futility can be interpreted as a lack of communication between the physician and the patient or relatives. In the article, Futility: What Cool Hand Luke Should Teach the Surgical Community, the authors cite the protagonist in the movie Cool Hand Luke tells the warden in the last minutes before execution, "What we've got here is a failure of communication". The authors of this article recommend and say, "Rather than refer to treatment options as futile, the term medical and surgically inappropriate should be invoked". "Increased communication and patient relations must be improved". Other authors question why the term futility is still in use in medicine and then recommend its elimination from medicine [4]. The term hanging the crepe has been used in medicine to describe the darkness possible outcome in the course of the disease in a particular patient. The term is totally pessimistic, leaving no space for recovery [1].

Blaise Pascal, a 17th century philosopher, who lived in a time of great skepticism. He stated that it is better than not to believe in God. Even if there is no certainty, no proof, no guarantee you should bet for the existence of God. Pascal's wager is a strategy of optimism and affirmation, while hanging the crepe is one of pessimism [1]. It is better to take the risk, even when the odds for success are low, when there is nothing to lose and a lot to gain $[5,6]$. The Pascal's Wager is very much alike to making a decision to operate on a very high risk patient, that some think is an act of futility. The mortality will be $100 \%$ without operation: but surgery is the only option available to obtain good results, though slim. The surgeon should always transmit optimism to the patient; in my two cases dealing with the relatives, since the patients were totally disconnected from reality.

And when there is no hope since the operative findings are not amenable for removal for cure or palliation, utmost care must be taken how to convey the findings but leaving a small window of hope. This will prevent radical decisions from the part of the patient including suicide. The word futility should never be used in the physician-patient relationship, nor to the relatives. The expression, there is no hope beyond this point as in Dante's Inferno should never be used. The word futility should be used by the physicians to decide the best course of action in a given patient based on the disease and the natural history of the disease in question and based on his/her past experience or the medical literature. The behavior of the physicians and particularly the surgeon should be the middle road as promoted by the philosopher René Descartes.

These two patients presented advanced neurological symptoms secondary to severe hypercalcemia such as loss of memory, coma and bony pains. Removal of parathyroid adenomas resulted in recovery of mental function, normal calcium and PTH levels. They are enjoying normal lives six and five years after surgery. It was more productive to take the risk of operation although the chances of success were low.

\section{Conclusion}

Two patients with hypercalcemia and severe neurological symptoms are presented. They had disorientation, coma, bone pain and decubitus ulcers. At surgery they had a single large parathyroid adenoma. After removal of the adenomas the serum calcium and PTH levels returned to normal and the patients recovered regaining their mental function. Both had been classified as high risk patients. After discussing the risks versus the benefits of the operation their respective families agreed to the surgical procedure. They are having normal lives six and five years after the operation.

\section{References}

1. Siegler M (1975) Pascal's Wager and the hanging of crepe. N Engl J Med 293(17): 853-857.

2. Dotzenrath CM, Kaetsch AK, Pfingsten H, Cupisti $\mathrm{K}$, Weyerbrock N, et al. (2006) Neuropsychiatric and cognitive changes after surgery for primary Hyperparathyroidism. World J Surg 30(5): 680-685.

3. Karpati G, Frame B (1964) Neuropsychiatric disorders in primary hyperparathyroidism Clinical analysis with review of the literature. JAMA Neurol 10(4): 387-397.

4. Grossman E, Angelos P (2009) Futility: what Cool Hand Luke Can Teach the Surgical Community. World J Surg 33(7): 1338-1340.

5. Papineau D (2009) Philosophy. Oxford University Press, pp: 121.

6. Ehrman BD (2014) How Jesus Became God. Harper Collins Publishers, pp: 283-284. 